\title{
TIME-DEPENDENT PHOTODISSOCIATION REGIONS
}

\author{
David Hollenbach
}

MS 245-3, NASA Ames Research Center, Moffett Field, CA 94035

AND

ANTONElla NatTa

Osservatorio Astrofisico di Arcetri, Firenze, Italy

Received 1995 March 27; accepted 1995 June 20

\begin{abstract}
We present theoretical models of the time-dependent thermal and chemical structure of molecular gas suddenly exposed to far-ultraviolet (FUV) $(6 \mathrm{eV}<h v<13.6 \mathrm{eV})$ radiation fields and the consequent timedependent infrared emission of the gas. We focus on the response of molecular hydrogen for cloud densities ranging from $n=10^{3}$ to $10^{6} \mathrm{~cm}^{-3}$ and FUV fluxes $G_{0}=10^{3}-10^{6}$ times the local FUV interstellar flux. For $G_{0} / n>10^{-2} \mathrm{~cm}^{3}$, the emergent $\mathrm{H}_{2}$ vibrational line intensities are initially larger than the final equilibrium values. The $\mathrm{H}_{2}$ lines are excited by FUV fluorescence and by collisional excitation in warm gas. Most of the $\mathrm{H}_{2}$ intensity is generated at a characteristic hydrogen column density of $N \sim 10^{21} \mathrm{~cm}^{-2}$, which corresponds to an FUV optical depth of unity caused by dust opacity. The time dependence of the $\mathrm{H}_{2}$ intensities arises because the initial abundances of $\mathrm{H}_{2}$ at these depths is much higher than the equilibrium values, so that $\mathrm{H}_{2}$ initially competes more effectively with dust in absorbing FUV photons. Considerable column densities of warm $(T \sim 1000) \mathrm{K} \mathrm{H}_{2}$ gas can be produced by the FUV pumping of $\mathrm{H}_{2}$ vibrational levels followed by collisional de-excitation, which transfers the energy to heat. In dense $\left(n \gtrsim 10^{5} \mathrm{~cm}^{-3}\right)$ gas exposed to high $\left(G_{0} \gtrsim\right.$ $10^{4}$ ) fluxes, this warm gas produces a $2-1 S(1) / 1-0 S(1) \mathrm{H}_{2}$ line ratio of $\sim 0.1$, which mimics the ratio found in shocked gas. In lower density regions, the FUV pumping produces a pure-fluorescent ratio of $\sim 0.5$. We also present calculations of the time dependence of the atomic hydrogen column densities and of the intensities of $\mathrm{O}_{\text {I }} 6300 \AA, \mathrm{S}$ II $6730 \AA, \mathrm{Fe}$ II $1.64 \mu \mathrm{m}$, and rotational $\mathrm{OH}$ and $\mathrm{H}_{2} \mathrm{O}$ emission. Potential applications include star-forming regions, clouds near active galactic nuclei, and planetary nebulae. We apply our models to five planetary nebulae and conclude that only $\mathrm{BD}+30^{\circ} 3639$ shows evidence of enhanced $\mathrm{H}_{2}$ emission due to (high) nonequilibrium $\mathrm{H}_{2}$ abundances.
\end{abstract}

Subject headings: infrared: ISM : lines and bands - ISM : clouds - ISM : molecules planetary nebulae: general — ultraviolet: ISM

\section{INTRODUCTION}

The study of photodissociation regions (PDRs) is essential in understanding the properties of the interstellar medium. PDRs are neutral regions where far-ultraviolet (FUV) ( $6 \mathrm{eV}<h v<13.6 \mathrm{eV}$ ) photons (generally from OB stars or white dwarfs) play a significant role in the heating or chemistry of the gas. By this definition, PDRs include most of the mass of the interstellar medium, not only the atomic but also the molecular regions, where all the hydrogen is in $\mathrm{H}_{2}$ and all the carbon is in $\mathrm{CO}$ but where residual FUV photons dominate the gas heating or the photodissociation of other important molecules, such as $\mathrm{O}_{2}$ or $\mathrm{H}_{2} \mathrm{O}$. PDRs produce much of the continuum and line infrared emission in most normal or starburst galaxies, for it is here that much of the starlight is absorbed by dust and where a fraction of the starlight energy is transformed to gas heating (Tielens \& Hollenbach 1985, hereafter TH85; Sternberg \& Dalgarno 1989; Hollenbach, Takahashi, \& Tielens 1991, hereafter HTT91). Genzel (1991, 1992) recently reviewed the extensive observations of PDRs in the Milky Way as well as in many other galaxies.

Most theoretical models of PDRs have focused on equilibrium chemical solutions (see Hollenbach \& Tielens 1995 for a recent review of theoretical models). However, there has been a small number of time-dependent PDR calculations discussed in the last two decades. Hill \& Hollenbach (1978) examined the propagation of the $\mathrm{H} / \mathrm{H}_{2}$ dissociation front when an $\mathrm{O}$ star suddenly turns on in a molecular cloud of density $n=10^{3}-10^{4}$ $\mathrm{cm}^{-3}$, where $n=n_{\mathrm{H}}+2 n_{\mathrm{H}_{2}}$ is the hydrogen nucleus density. A prime concern was whether the shock associated with the expanding $\mathrm{H}$ II region propagates into molecular or atomic gas. London (1978) studied the nonequilibrium $\mathrm{H}_{2}$ front established when, for example, an OB star moves through a molecular cloud. Roger \& Dewdney (1992) extended the work of Hill \& Hollenbach by including heating and cooling and expanding the parameter space to include densities of $10^{1}-10^{4} \mathrm{~cm}^{-3}$ and a range of stellar effective temperatures. Goldshmidt \& Sternberg (1995, hereafter GS95) recently studied the onedimensional $\mathbf{H} / \mathbf{H}_{2}$ dissociation wave propagating through a semi-infinite molecular slab that is suddenly exposed to an FUV flux $G_{0}$. Their focus was on the time-dependent fluorescent $\mathrm{H}_{2}$ emission produced for densities $n=10^{2}-10^{4} \mathrm{~cm}^{-3}$ and $G_{0}=10^{\circ}-10^{6}$, in units of the local interstellar FUV flux.

Time-dependent PDR calculations are interesting because the timescales to establish $\mathrm{H} / \mathrm{H}_{2}$ equilibrium, $t_{\mathrm{cq}} \sim 5 \times 10^{8}$ $n^{-1} \mathrm{yr}$, are often long compared with the timescales for variation in the FUV field or the gas density. For example, we are primarily motivated by the PDRs created in the dense $\left(n \leqslant 10^{6}\right.$ $\mathrm{cm}^{-3}$ ) neutral shells around planetary nebulae. Here the central star may "turn on "its FUV luminosity in $\sim 10^{2}-10^{3}$ $y r$, and the density and FUV flux change on the shell dynamical timescale of $\sim 10^{3} \mathrm{yr}$. Other applications include starforming regions and clouds near active galactic nuclei (AGNs), 
where clumpy material can be suddenly exposed to strong FUV radiation as clumps move out of shadowed regions.

We present here a parameter study of constant-density, $n=10^{3}-10^{6} \mathrm{~cm}^{-3}$, semi-infinite slabs of molecular gas exposed at $t=0$ to an FUV flux $G_{0}=10^{3}-10^{6}$. The focus is on the time-dependent photodissociation and fluorescence of $\mathrm{H}_{2}$ and the heating that accompanies the rapid FUV pumping and dissociation of the $\mathrm{H}_{2}$ molecules. The relatively high densities lead to appreciable collisional de-excitation of the FUVpumped, vibrationally excited $\mathrm{H}_{2}$, and the heating leads, in several cases, to a substantial thermal component to the $\mathrm{H}_{2}$ 1-0 $S(1)$ and 2-1 $S(1)$ intensities, thereby modifying the purefluorescent spectrum. We also calculate the atomic hydrogen column densities and the intensities of other observable lines that show interesting time dependence, including rotational transitions of $\mathrm{OH}$ and $\mathrm{H}_{2} \mathrm{O}$ and the forbidden [ $\left.\mathrm{Fe} \mathrm{II}\right] 1.64 \mu \mathrm{m}$, [O I] $6300 \AA$, and [S II] $6730 \AA$. This work complements the results of GS 95 by extending the parameter space to higher densities and by including a solution to the thermal balance and the line emission from a number of species.

The paper is organized as follows: In $\S 2$ we describe the physical processes incorporated into the time-dependent PDR code. In $\S 3$ we present detailed results for a specific case $\left(n=10^{5} \mathrm{~cm}^{-3}\right.$ and $\left.G_{0}=10^{5}\right)$ and discuss simple analytic approximations to the evolution of the PDR. We also present and discuss the atomic hydrogen column densities and the emergent line intensities as a function of $n, G_{0}$, and $t$. We briefly discuss possible applications of our general models in $\$ 4$ and summarize our conclusions in $\S 5$. We discuss the numerical methods in the Appendix. We have incorporated this timedependent PDR code in a much more comprehensive code designed to follow the evolution of expanding neutral shells or clumps surrounding planetary nebulae; these results will be presented in a subsequent paper (Natta \& Hollenbach 1995).

\section{PHYSICAL PROCESSES AND NUMERICAL METHODS}

\subsection{Chemistry and Timescales}

The neutral hydrogen is initially entirely molecular, but the onset of the FUV flux sends a wave of dissociation into the neutral layer. The time-dependent equation for the molecular hydrogen density $n_{\mathrm{H}_{2}}$ is

$$
\frac{d n_{\mathrm{H}_{2}}}{d t}=R_{f} n n_{\mathrm{H}}-G_{0} I_{0} f\left(N_{\mathrm{H}_{2}}\right) e^{-\sigma \mathrm{FuVN}} n_{\mathrm{H}_{2}},
$$

where $R_{f}=3 \times 10^{-17} \mathrm{~cm}^{3} \mathrm{~s}^{-1}$ is the rate coefficient for $\mathrm{H}_{2}$ formation on grains, $n_{\mathrm{H}}$ and $n_{\mathrm{H}_{2}}$ are the atomic and molecular hydrogen densities, $I_{0}=4 \times 10^{-11} \mathrm{~s}^{-1}$ is the unattenuated $\mathrm{H}_{2}$ photodissociation rate in the local interstellar radiation field, $G_{0}$ is the FUV photon flux incident on the slab in units of the local interstellar radiation field (about $2 \times 10^{7}$ photons $\mathrm{cm}^{-2}$ $\mathrm{s}^{-1}$ for the range $\left.11 \mathrm{eV}<h v<13.6 \mathrm{eV}\right), N$ and $N_{\mathrm{H}_{2}}$ are the hydrogen nucleus and molecular hydrogen column densities, $f\left(N_{\mathrm{H}_{2}}\right)$ is the self-shielding factor for $\mathrm{H}_{2}$ (GS95), and $\sigma_{\mathrm{FuV}}=1.4$ $\times 10^{-21} \mathrm{~cm}^{-2}$ is the effective FUV grain-absorption cross section per hydrogen nucleus (TH85; GS95). In equation (1), the first term is the formation rate of $\mathrm{H}_{2}$ on grains per unit volume; the second term is the FUV photodissociation rate of $\mathrm{H}_{2}$ per unit volume. At sufficiently high molecular column densities, $N_{\mathrm{H}_{2}} \gtrsim 10^{15} \mathrm{~cm}^{-2}$, FUV absorption in the Lorentzian wings of the Lyman-Werner lines initiates dissociation. The self-shielding factor then becomes $f\left(N_{\mathrm{H}_{2}}\right)=\beta / N_{\mathrm{H}_{2}}^{1 / 2}$, where $\beta \simeq 10^{6} \mathrm{~cm}^{-1}$ (Jura 1974; J. Black 1995, private communication). This condition generally applies for most regions of interest in the dissociation wave, as we shall discuss below. The characteristic timescale for $\mathrm{H}_{2}$ to reach equilibrium is given, from equation (1), by (see also GS95)

$$
t_{\mathrm{eq}}=\frac{1}{2 R_{f} n} \simeq 500 \mathrm{yr}\left(\frac{10^{6} \mathrm{~cm}^{-3}}{n}\right) .
$$

It should be noted that $t_{\mathrm{eq}}$ is the timescale to reach equilibrium deep in the slab, where the fractional abundances of $\mathrm{H}$ and $\mathrm{H}_{2}$ are comparable $\left(x_{\mathrm{H}} \sim x_{\mathrm{H}_{2}}\right)$ and the FUV photodissociation is heavily shielded by dust and/or hydrogen molecules. As we shall discuss below, if this occurs at $N>\sigma_{\text {Fuv }}^{-1} \sim 10^{21} \mathrm{~cm}^{-2}$, then this is also the timescale for the $\mathrm{H}_{2}$ fluorescent intensities to drop from high, nonequilibrium values to much lower, equilibrium values. However, in the relatively unshielded regions where the $\mathrm{H}_{2}$ equilibrium abundance $x_{2}^{\text {eq }} \ll 1$, the equilibrium timescale is much shorter than $t_{\mathrm{eq}}$ and scales inversely with $G_{0}$. We emphasize that our focus is on the parameter space $G_{0} / n>10^{-2} \mathrm{~cm}^{3}$, so that the equilibrium abundance of $\mathrm{H}_{2}$ is very low until $N>10^{21} \mathrm{~cm}^{-2}$, when dust shielding aids selfshielding and results in primarily molecular gas.

In order to streamline the PDR code so that it may be used in our more complicated planetary nebula code (Natta \& Hollenbach 1995), we have made simple approximations for the chemistry of other potential gas coolants. Our main goal is to calculate the emergent intensities of the important cooling transitions C II $158 \mu \mathrm{m}, \mathrm{O}_{\text {I }} 63 \mu \mathrm{m}, \mathrm{Si}$ II $35 \mu \mathrm{m}$, Fe II $1.28,1.64$ $\mu \mathrm{m}, \mathrm{O}_{\mathrm{I}} 6300 \AA$, and $\mathrm{S}$ II $6730 \AA$ and the $\mathrm{OH}$ and $\mathrm{H}_{2} \mathrm{O}$ rotational transitions, all of which may be important in the relatively warm and unshielded region $N<4 \times 10^{21} \mathrm{~cm}^{-2}$, where the $\mathrm{H}_{2}$ emission originates. We have assumed gas-phase abundances of the elements $x_{\mathrm{C}}=3 \times 10^{-4}, x_{\mathrm{O}}=5 \times 10^{-4}, x_{\mathrm{Si}}=$ $3.5 \times 10^{-5}, x_{\mathrm{S}}=10^{-5}$, and $x_{\mathrm{Fe}}=10^{-6}$. With the exceptions of $\mathrm{OH}$ and $\mathrm{H}_{2} \mathrm{O}$, we have used the equilibrium PDR results of TH85 and HTT91 to estimate the abundances $x\left(\mathrm{C}^{+}\right), x(\mathrm{CO})$, $x(\mathrm{O}), x\left(\mathrm{Si}^{+}\right), x\left(\mathrm{Fe}^{+}\right)$, and $x\left(\mathrm{~S}^{+}\right)$as a function of column density $N$. We justify below the use of equilibrium abundances for these species, describe our simple fit to the equilibrium results, and discuss the calculation of $\mathrm{OH}$ and $\mathrm{H}_{2} \mathrm{O}$ abundances.

The timescale for $\mathrm{CO}$ to photodissociate and for carbon to ionize to $\mathrm{C}^{+}$in the neutral PDR is given by the photodissociation time of $\mathrm{CO}$, which is prolonged by the selfshielding of $\mathrm{CO}$ but is nevertheless much shorter than the timescale for $\mathrm{H}_{2}$ dissociation because $N(\mathrm{CO}) \ll N_{\mathrm{H}_{2}}$. For hydrogen column densities $N \gtrsim 10^{21} \mathrm{~cm}^{-2}$, dust shielding sets in and $\mathrm{CO}$ persists. The maximum timescale required for $\mathrm{C} / \mathrm{C}^{+} / \mathrm{CO}$ equilibrium is therefore given by the self-shielded photodissociation rate, calculated at $N \simeq 10^{21} \mathrm{~cm}^{-2}$ with $N_{\mathrm{H}_{2}} \simeq 5 \times 10^{20} \mathrm{~cm}^{-2}$ and $N_{\mathrm{co}} \simeq 3 \times 10^{17} \mathrm{~cm}^{-2}$ (all carbon in $\mathrm{CO}$ and all hydrogen in $\mathrm{H}_{2}$ from the surface to $N=10^{21}$ $\mathrm{cm}^{-2}$ ). Using these maximal-shielding column densities (the $\mathrm{H}_{2}$ also partially shields the $\mathrm{CO}$ ), we derive, using shielded rates from van Dishoeck \& Black (1988), the carbon timescale

$$
t_{\mathrm{C}^{+}} \lesssim 0.8 \mathrm{yr}\left(\frac{10^{5}}{G_{0}}\right) .
$$

This timescale is about 2 orders of magnitude [roughly $\left(N_{\mathrm{Co}} / N_{\mathrm{H}_{2}}\right)^{1 / 2}$ ] smaller than the equivalent timescale for $\mathrm{H}_{2}$ photodissociation at the same column density. The timescale for other oxygen-bearing molecules, such as $\mathrm{O}_{2}, \mathrm{OH}$, and $\mathrm{H}_{2} \mathrm{O}$, to photodissociate is even shorter since self-shielding is 
unimportant:

$$
t_{0} \simeq 10^{-3} \mathrm{yr}\left(\frac{10^{5}}{G_{0}}\right) .
$$

These timescales are sufficiently short compared to the $\mathrm{H}_{2}$ timescales that we assume equilibrium PDR solutions apply for $\mathrm{CO}, \mathrm{C}^{+}$, and $\mathrm{O}$.

The equilibrium PDR results of TH85 and HTT91 show that for $G_{0} \gtrsim 10^{2}, n \gtrsim 10^{4} \mathrm{~cm}^{-3}$, and $G_{0} / n \gtrsim 10^{-3} \mathrm{~cm}^{3}$, the carbon is ionized to hydrogen column densities of $\sim 4 \times 10^{21}$ $\mathrm{cm}^{-2}$ and the oxygen is entirely atomic to this same column density. Beyond this column density the carbon is in $\mathrm{CO}$, and all oxygen not in CO remains predominantly atomic to $N \gtrsim$ $10^{22} \mathrm{~cm}^{-2}$. Similarly, sulfur is ionized to $N \simeq 4 \times 10^{21} \mathrm{~cm}^{-2}$, while iron is ionized to greater depths. We therefore assume this simple prescription for the abundances of these species.

However, inside the column density $N<4 \times 10^{21} \mathrm{~cm}^{-2}$, a small but significant fraction of the oxygen may be converted to $\mathrm{OH}$ and $\mathrm{H}_{2} \mathrm{O}$ by neutral-neutral reactions that proceed rapidly in hot molecular gas such as is produced when the dissociation wave propagates through the low column density gas. Although we find that the resultant enhanced abundances of $\mathrm{OH}$ and $\mathrm{H}_{2} \mathrm{O}$ do not dominate the cooling in the cases considered, we include the emission from these species as possible diagnostics of the time-dependent PDR. The important reactions include

$$
\mathrm{O}+\mathrm{H}_{2} \rightarrow \mathrm{OH}+\mathrm{H}, \quad \mathrm{OH}+\mathrm{H}_{2} \rightarrow \mathrm{H}_{2} \mathrm{O}+\mathrm{H} .
$$

The rate coefficients for these reactions and the reverse reactions are given, for example, in Hollenbach \& McKee (1989). We include in our PDR code this reaction sequence and the FUV photodissociation of $\mathrm{OH}$ and $\mathrm{H}_{2} \mathrm{O}$ (see Hollenbach \& McKee 1989 for FUV cross sections)

This approximate chemistry underestimates the production of $\mathrm{OH}$ and $\mathrm{H}_{2} \mathrm{O}$ because it ignores the ion-molecule reactions that can also produce enhanced $\mathrm{OH}$ and $\mathrm{H}_{2} \mathrm{O}$ abundances in regions where the $\mathrm{H}_{2}$ is higher than equilibrium. In addition, the enhanced $\mathrm{OH}$ abundance leads to an enhanced (though still small) abundance of $\mathrm{CO}$. The $\mathrm{CO}$ chemistry is sufficiently complicated that we do not calculate the (warm) $\mathrm{CO}$ abundances in the enhanced-OH region, and therefore we cannot predict the emergent $\mathrm{CO}$ spectrum in this paper. However, the ion-molecule enhancements to $\mathrm{OH}$ and $\mathrm{H}_{2} \mathrm{O}$ are small compared to their production by neutral-neutral reactions in the warm $(T>300 \mathrm{~K})$ regions, and the FUV photodissociation prevents $\mathrm{CO}$ from being the dominant coolant in these regions, so the overall temperature structure of the warm gas is well approximated. Our goal here is to calculate the $\mathrm{H}_{2}$ and atomic (C II $158 \mu \mathrm{m}, \mathrm{O}_{\text {I }} 63 \mu \mathrm{m}, \mathrm{Fe}_{\text {II }} 1.2,1.6 \mu \mathrm{m}, \mathrm{O}_{\text {I }} 6300 \AA$, and $\mathrm{S}$ II $6730 \AA$ ) intensities and to make (lower limit) estimates of the $\mathrm{OH}$ and $\mathrm{H}_{2} \mathrm{O}$ emission.

\subsection{Thermal Balance and Radiative Transfer}

The neutral gas is heated primarily by grain photoelectric heating (Bakes \& Tielens 1994) and the heating caused by FUV pumping and formation pumping of the hydrogen molecule, followed by collisional de-excitation of the excited vibrational levels (see TH85; Hollenbach \& McKee 1989). $\mathrm{H}_{2}$ photodissociation also is a heating agent (TH85). These processes are well known and are often applied in the context of equilibrium chemistry; the novel aspect of their application to timedependent PDRs is that, for example, nonequilibrium FUV pumping may dominate the heating as the dissociation wave sweeps through the molecular gas.

Significant coolants in the neutral region include vibrational and rotational transitions of $\mathrm{H}_{2}$, collisional dissociation of $\mathrm{H}_{2}$, rotational transitions of $\mathrm{CO}, \mathrm{OH}$, and $\mathrm{H}_{2} \mathrm{O}$, fine-structure transitions of $\mathrm{O}$ and $\mathrm{C}^{+}$, and grain cooling of the gas. We also include collisional excitation of $\mathrm{Ly} \alpha,\left[\mathrm{O}_{\mathrm{I}}\right] 6300 \AA$, [S II] 6730 $\AA$, and $[\mathrm{Fe}$ II $] 1.28,1.64 \mu \mathrm{m}$, which are appreciable when the neutral region is driven to $T \gtrsim 3 \times 10^{3} \mathrm{~K}$. The cooling rates for these processes have been taken from Hollenbach \& McKee $(1979,1989)$ and TH85. The O I $63 \mu \mathrm{m}, C_{\text {II }} 158 \mu \mathrm{m}$, $\mathrm{CO}, \mathrm{OH}$, and $\mathrm{H}_{2} \mathrm{O}$ lines are treated with the escapeprobability formalism described in Hollenbach \& McKee (1979) because their line opacities can be large and selfabsorption followed by collisional de-excitation may be important. A cooling time can be estimated by assuming LTE cooling by $\mathrm{H}_{2}$ :

$$
t_{c} \sim 4 T_{3}^{-7 / 2} \mathrm{yr},
$$

where $T_{3}=T / 1000 \mathrm{~K}$ and we have fitted the numerical cooling results of Hollenbach \& McKee (1979) with a power law valid over the range $0.3 \leqslant T_{3} \leqslant 3$. This timescale is sufficiently short that we assume thermal equilibrium while we calculate the nonequilibrium evolution of the hydrogen chemistry. On the other hand, the dissociation front moves rapidly through the cloud and causes a brief temperature spike in the gas at the dissociation front. We have checked the timescales and find that thermal equilibrium remains a reasonable assumption, even in the spike, for most cases of interest. In any event, there is little contribution to the overall observable line intensities from the hot spike, as discussed in $\S 3.3$.

The emergent intensities in the lines are found by integrating the escaping line emissivity through the thickness of the slab and dividing by $4 \pi$. For the case of the vibrational emission from $\mathrm{H}_{2}$, we add to the thermal component a "nonthermal" contribution to the 1-0 $S(1)$ and 2-1 $S(1)$ intensities caused by the FUV pumping and formation pumping of the vibrational states. At high densities, $n>n_{\mathrm{cr}}$, the pumping contributions are decreased by a factor $n_{\mathrm{cr}} / n$, where $n_{\mathrm{cr}}$ is the characteristic critical density for the collisional de-excitation of an excited vibrational level (see Burton, Hollenbach, \& Tielens 1990 for details of this procedure).

\section{MODEL RESULTS}

\subsection{The Time Evolution of the Dissociation Front}

Figures 1 and 2 present some detailed results for the evolution of a slab with $G_{0}=10^{5}$ and $n=10^{5} \mathrm{~cm}^{-3}$. We use this case to demonstrate the evolution of the $\mathrm{H}_{2}$ dissociation front and to examine in detail the temperature structure and the dominant heating and cooling processes in the gas.

The evolution of $x_{\mathrm{H}_{2}}$ as a function of $N$ and $t$ is shown in the bottom panel of Figure 1. Note that $N$, the hydrogen nucleus column density, is linearly related to depth $l$ into the slab, $l=N / n$. At early times, a steep dissociation front is apparent, where $x_{\mathrm{H}_{2}}$ rises from its low equilibrium value $\left(\lesssim 10^{-3}\right)$ to high, substantially molecular values $(\gtrsim 0.1)$ at a column density $N^{*}$ We define $N^{*}$ to be the column density where the gas is halfatomic $\left(x_{\mathrm{H}}=0.5\right)$ and half-molecular $\left(x_{\mathrm{H}_{2}}=0.25\right)$. For these values of $G_{0}$ and $n, N^{*}<10^{21} \mathrm{~cm}^{-2}$ for $t \leqslant 100 \mathrm{yr}$ and grain attenuation is negligible at the front. Figure 1 shows that for these early times $N^{*} \propto t^{2}$, or the velocity of the front $v_{D} \propto$ $d N^{*} / d t \propto t$. For $t \gtrsim 100 \mathrm{yr}$, the front reaches $N^{*} \gtrsim \sigma_{\mathrm{FUV}}^{-1} \sim$ 

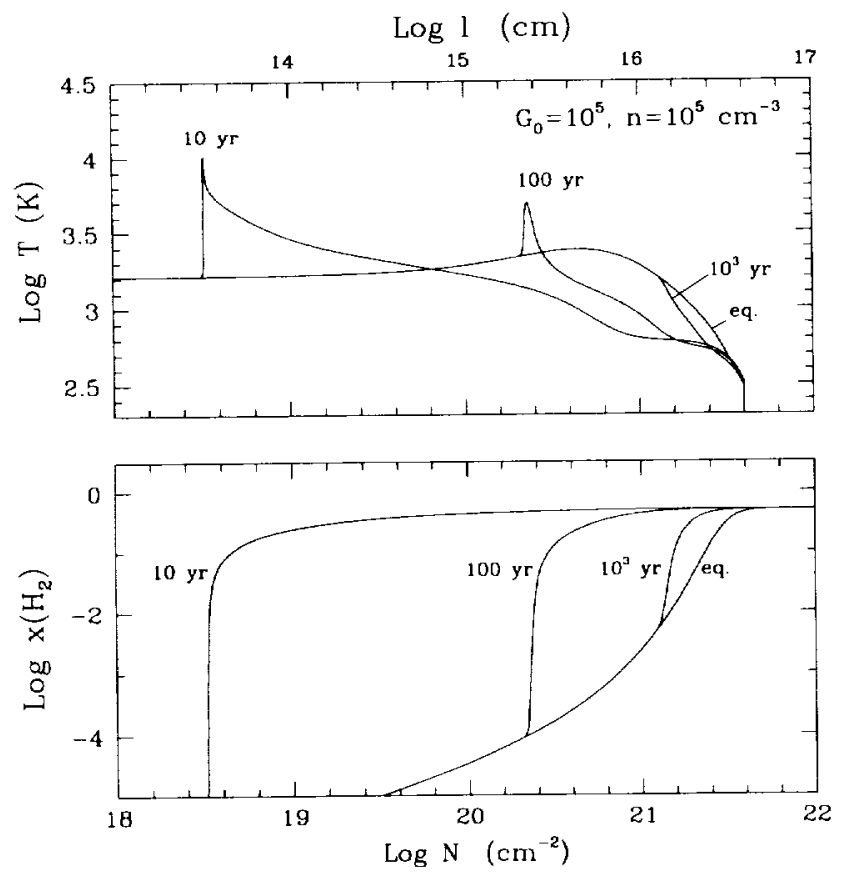

FiG. 1.- Temperature $T$ (top) and $\mathrm{H}_{2}$ fractional abundance $x_{\mathrm{H}_{2}}$ (bottom) piotted as functions of the gas column density in the slab at different times. The slab has $G_{0}=10^{5}, n=10^{5} \mathrm{~cm}^{-3}$. The depth into the slab $l$ is also shown on the top scale. The steep dissociation front initially accelerates $\left(v_{D} \propto t\right)$ into the slab. Enhanced column densities of warm $\mathrm{H}_{2}$ are evident at early times.

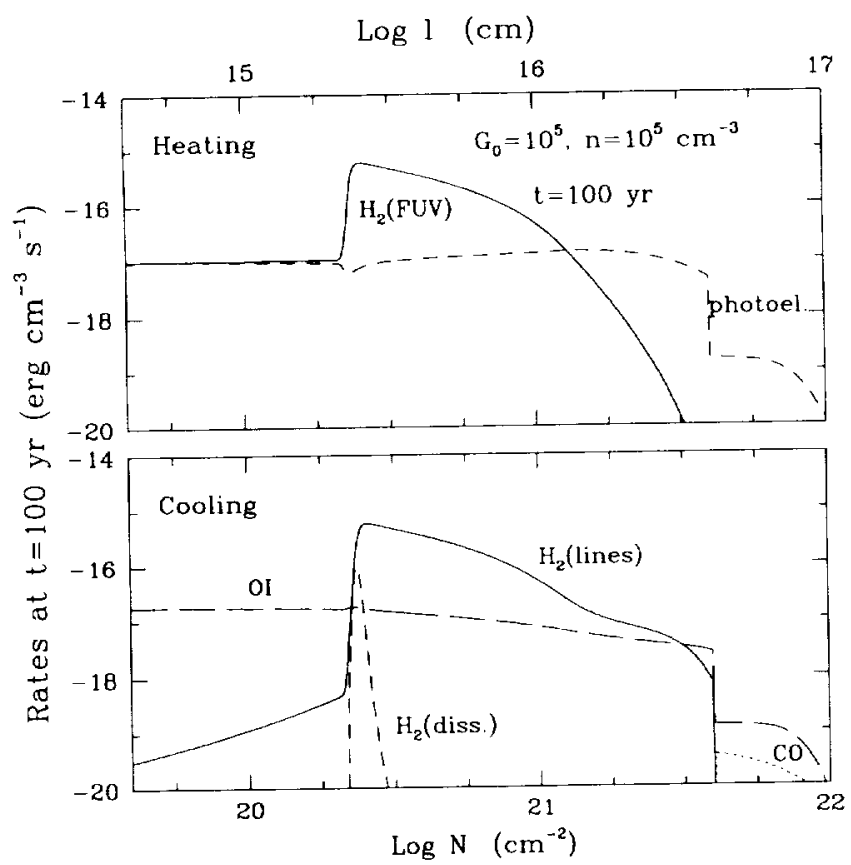

FIG. 2.-Heating and cooling rates of the dominant processes shown as functions of the gas column density at $t=100$ yr for a slab with $G_{0}=10^{5}$, $n=10^{5} \mathrm{~cm}^{-3}$. The upper panel plots heating rates; the solid line is the heating due to FUV pumping of $\mathbf{H}_{2}$ followed by collisional de-excitation, the dashed line the heating due to photoelectric effect on grains. The lower panel plots cooling rates; the solid line shows the rate of cooling due to $\mathrm{H}_{2}$ rotational and vibrational line emission, the short-dashed line the rate of cooling due to collisional dissociation of $\mathrm{H}_{2}$, the long-dashed line the $\mathrm{O} / 63 \mu \mathrm{m}$ cooling, and the dotted line the cooling due to $\mathrm{CO}$ lines.
$10^{21} \mathrm{~cm}^{-2}$, grain attenuation lowers the FUV flux, and the front slows and eventually stops as equilibrium is reached at $t \gtrless 10^{3} \mathrm{yr}$.

The steepness of the front is caused by "runaway" dissociation at small column densities. The self-shielding causes the highest dissociation rates to occur at the smallest $N$. As molecules are dissociated at low $N$, the self-shielding decreases further, so the (already high) dissociation rates appreciably increase with time at low $N$, whereas at high $N$ the shielding column densities have not substantially changed $\left(N_{\mathbf{H}_{2}} \sim 0.5 N\right)$ and the dissociation rates are constant in time.

Ignoring dust extinction (i.e., $N<10^{21} \mathrm{~cm}^{-2}$ ), one can analytically solve equation (1) and derive the $N$ and $t$ dependence of $x_{\mathrm{H}}$ and $x_{\mathrm{H}_{2}}$ for the region $N>N^{*}$ since, at these columns, $N_{\mathrm{H}_{2}} \simeq 0.5 N$ :

$$
\begin{gathered}
x_{\mathrm{H}}=2^{1 / 2} \beta I_{0} G_{0} N^{-1 / 2} t, \\
x_{\mathrm{H}_{2}}=0.5\left(1-x_{\mathrm{H}}\right),
\end{gathered}
$$

valid for

$$
N^{*}<N<\sigma_{\text {FUV }}^{-1} \sim 10^{21} \mathrm{~cm}^{-2} .
$$

$N^{*}<N$ is the equivalent to the condition

$$
t<\left(2^{1 / 2} \beta I_{0} G_{0} N^{-1 / 2}\right)^{-1} \sim 180 N_{21}^{1 / 2} G_{05}^{-1} \mathrm{yr},
$$

where $N_{21}=N / 10^{21} \mathrm{~cm}^{-2}$ and $G_{05}=G_{0} / 10^{5}$. Equations (6) and (7) also assume that $N>10^{15} \mathrm{~cm}^{-2}$, so the self-shielding factor $f\left(N_{\mathrm{H}_{2}}\right)=\beta(0.5 N)^{-1 / 2}$, where $\beta=10^{6} \mathrm{~cm}^{-1}$ (see $\S 2.1$ ). Either equation (6) or equation (7) can be used to derive $N^{*}(t)$, since the front tracks the position $x_{\mathrm{H}_{2}} \sim 0.25$ or $x_{\mathrm{H}} \sim 0.5$ :

$$
N^{*}(t) \simeq 8 \beta^{2} I_{0}^{2} G_{0}^{2} t^{2} \simeq 1.2 \times 10^{21} G_{05}^{2} t_{2}^{2} \mathrm{~cm}^{-2},
$$

where $t_{2}=t / 10^{2} \mathrm{yr}$. We note that $N^{*}$ is greater than the column density where the front becomes extremely steep (see Fig. 1); this is because $N^{*}$ is defined as the column density to $x_{\mathbf{H}} \sim 0.5$, whereas the front steepens at $x_{H} \sim 0.1$. The front velocity is calculated from equation (8) as

$$
v_{D} \simeq 16 \beta^{2} I_{0}^{2} G_{0}^{2} n^{-1} t \simeq 77 G_{05}^{2} n_{5}^{-1} t_{2} \mathrm{~km} \mathrm{~s}^{-1}
$$

where $n_{5}=n / 10^{5} \mathrm{~cm}^{-3}$. Equation (8) and (9) are valid for $N^{*}<\sigma_{\text {fuv }}^{-1} \sim 10^{21} \mathrm{~cm}^{-2}$ or, equivalently, $t<t_{\sigma}$, where $t_{\sigma}$ is the time for the front to reach $N=\sigma_{\mathrm{FUv}}^{-1}$ :

$$
t_{\sigma}=\left(8 \beta^{2} I_{0}^{2} G_{0}^{2} \sigma_{\mathrm{FUv}}\right)^{-1 / 2} \simeq 79 G_{05}^{-1} \mathrm{yr} .
$$

Equations (6)-(9) also implicitly assume that $x_{\mathrm{H}_{2}}$ is significantly greater than its equilibrium value in the region of interest. This is always true for $N<10^{21} \mathrm{~cm}^{-2}$ and $t<t_{\sigma}$ if $G_{0} / n>10^{-2} \mathrm{~cm}^{3}$ (see $\left.\$ 2.1\right)$.

\subsection{Comparison of $v_{D}$ with Previous Results}

Equation (9) seems to contradict the result in GS 95, where the "dissociation front" had constant speed with time. However, the discrepancy is only caused by a difference in the definition of the dissociation front. In GS95 the column density $N$ to the front is identified as $N_{\mathrm{M}}^{\mathrm{tot}}$, the total (i.e., integrated to infinite depth) column density of atomic hydrogen at time $t$. By our definition, the column density to the front is $N^{*}$, the column density at which the local abundances are halfmolecular and half-atomic. These two column densities are different and have different time dependencies. Note from equation (6) that $N_{\mathrm{H}} \propto x_{\mathrm{H}} N^{1 / 2}$, so $N_{\mathrm{H}}^{\text {tot }}$ is dominated by the highest column density gas, where dust does not appreciably attenuate the FUV (in this case $N \sim \sigma_{\text {FUV }}^{-1} \sim 10^{21} \mathrm{~cm}^{-2}$ for 
larger column densities grain attenuation of the FUV rapidly lowers $x_{H}$ ). In other words, if we use Figure 1 at $t=10 \mathrm{yr}$ as an example, it is not the completely dissociated $N^{*} \simeq 10^{19} \mathrm{~cm}^{-2}$ that dominates $N_{\mathrm{H}}^{\text {tot }}$ but the almost completely molecular gas at $N \sim 10^{21} \mathrm{~cm}^{-2}$, where $x_{\mathrm{H}} \sim 0.1$ and $N_{\mathrm{H}} \sim 7 \times 10^{19} \mathrm{~cm}^{-2}$. Therefore, we are in agreement with GS95 that $N_{\mathrm{H}}^{\text {tot }} \propto t$ at early times. From equation (6), taking the maximum $N$ to be $\sigma_{\mathrm{FuV}}^{-1}$, we obtain

$$
N_{\mathrm{H}}^{\mathrm{tot}} \simeq 2^{3 / 2} \beta I_{0} G_{0} \sigma_{\mathrm{FUV}}^{-1 / 2} t
$$

With the GS95 definition of the dissociation front, the front velocity is proportional to $d N_{\mathrm{H}}^{\text {tot }} / d t$, which is constant in time. However, for reasons made clear by Figure 1, we prefer our definition of the dissociation front, and we obtain $v_{D} \propto t$ for early times, $t<t_{\sigma}$ (or $N^{*}<10^{21} \mathrm{~cm}^{-2}$ ), and for $x_{\mathrm{H}_{2}}$ at $N^{*}$ significantly greater than the equilibrium value.

\subsection{Thermal Balance in Time-dependent PDRs}

Figure 2 illustrates some typical features of the thermal balance for the case $n=10^{5} \mathrm{~cm}^{-3}$ and $G_{0}=10^{5}$. The rapid rate per unit volume of FUV pumping of $\mathrm{H}_{2}$ at the front, where $x_{\mathbf{H}_{2}}$ is much larger than equilibrium and where there is little self-shielding, causes a temperature spike, as is evidenced by Figure 1. Figure 2 shows that, for the case studied, the heating in the spike is mainly caused by FUV pumping of $\mathrm{H}_{2}$ followed by collisional de-excitation. The cooling is by collisional dissociation of $\mathrm{H}_{2}$ and collisional excitation of ro-vibrational infrared lines of $\mathrm{H}_{2}$.

In the completely photodissociated region $N<N^{*}$, grain photoelectric heating and $\mathrm{H}_{2}$ FUV pump-heating are balanced by $\mathrm{O}$ I $63 \mu \mathrm{m}$ cooling to produce a temperature of about 1500 $\mathrm{K}$ in the atomic gas. Figure 1 also shows that the temperature at $N \sim 10^{21} \mathrm{~cm}^{-2}$ rises with time, which is caused by the decrease in the abundance of the coolant $\mathrm{H}_{2}$. At high column densities, $N>4 \times 10^{21} \mathrm{~cm}^{-2}$, the grain photoelectric heating suddenly drops (Fig. 2) because the approximate carbon chemistry produces a sudden drop in the electron abundance in the gas (which raises the positive grain charge and makes photoejection of electrons more difficult). Cooling is dominated here by $\mathrm{O}$ I $63 \mu \mathrm{m}$ and rotational transitions of $\mathrm{CO}$.

\subsection{FUV Pumping of $\mathrm{H}_{2}, \mathrm{H}_{2}$ Fluorescence, and Thermal $\mathrm{H}_{2}$ Emission}

GS95 extensively discuss $\mathrm{H}_{2}$ fluorescence in low-density $\left(n<n_{\mathrm{cr}} \sim 10^{4} \mathrm{~cm}^{-3}\right.$ ), time-dependent PDRs, and we briefly paraphrase their results here, with a somewhat different approach to explaining the limiting solutions. We first describe the low-density results, $n<n_{\mathrm{cr}}$, where collisional de-excitation of vibrationally excited $\mathrm{H}_{2}$ is negligible and a "purefluorescent" spectrum emerges.

Consider fluorescence when $N_{\mathrm{H}_{2}} \simeq 0.5 N$ at $N=\sigma_{\mathrm{FUV}}^{-1} \simeq$ $10^{21} \mathrm{~cm}^{-2}$. This case applies at all times for $G_{0} / n \lesssim 10^{-2} \mathrm{~cm}^{3}$ since self-shielding of $\mathrm{H}_{2}$ enables the equilibrium abundance of $\mathrm{H}_{2}$ to be $x_{\mathrm{H}_{2}} \sim 0.5$ at $N=10^{21} \mathrm{~cm}^{-2}$ when the FUV-todensity ratio is this low. It also applies at early times, $t<t_{\sigma}$ (or $N^{*}<10^{21} \mathrm{~cm}^{-2}$ ), for time-evolving PDRs with $G_{0} / n>10^{-2}$ $\mathrm{cm}^{3}$. The column pump rate $P_{\text {FUV }}$ (or the pump rate per unit PDR surface area) is about 9 times the column photodissociation rate of $\mathrm{H}_{2}$, or about 4.5 times the production rate of $\mathbf{H}$ :

$$
P_{\mathrm{FUV}} \simeq 4.5 \frac{d N_{\mathrm{H}}^{\mathrm{tot}}}{d t} \simeq 4.5\left(2^{3 / 2} \beta I_{0} G_{0} \sigma_{\mathrm{FUV}}^{-1 / 2}\right)
$$

where we have made use of equation (11). The total intensity $I_{1 \mathrm{R}}$ in the infrared lines in this case is given by

$$
I_{\mathrm{IR}}=I_{\sigma}=\frac{P_{\mathrm{FUV}} \Delta E_{p}}{4 \pi} \simeq 0.4 G_{05} \operatorname{ergs~cm}^{-2} \mathrm{~s}^{-1} \mathrm{sr}^{-1}
$$

where $\Delta E_{p}$ is the average ro-vibrational energy delivered per FUV pump ( $\sim 2.6 \mathrm{eV})$. Equation (13) can be easily understood. Essentially, $\mathrm{H}_{2}$ wins the competition with dust for FUV photons, and most of the photons in the energy range 11-13.6 $\mathrm{eV}$ are absorbed by $\mathrm{H}_{2}$ and give rise to excitation and fluorescence. In pure fluorescence the intensity of the 1-0 S(1) line is about $2 \%$ of $I_{1 \mathrm{R}}$ (see, e.g., GS95). The main result is that $I_{\mathrm{IR}}$ is constant $\left(=I_{\sigma}\right)$ at all times for $G_{0} / n \leqslant 10^{-2} \mathrm{~cm}^{3}$ and at early times, $t \lesssim t_{\sigma}$, for $G_{0} / n \gtrsim 10^{-2} \mathrm{~cm}^{3}$. Like the column density of atomic hydrogen, most of the line intensity originates from $N \sim \sigma_{\mathrm{FUV}}^{-1} \sim 10^{21} \mathrm{~cm}^{-2}$.

Now consider later times for the case $G_{0} / n \gtrsim 10^{-2} \mathrm{~cm}^{3}$. The gas becomes atomic at $N \sim 10^{21} \mathrm{~cm}^{-2}$. For $t_{\sigma} \lesssim t \lesssim t_{\text {eq }}$, the dissociation front slows as $N^{*}$ exceeds $\sigma_{\mathrm{Fuv}}^{-1}$ and the grains attenuate the FUV. Most of the dissociation and, therefore, most of the pumps occur between the front $N^{*}$ and $N^{*}+\sigma_{\mathrm{FUV}}^{-1}$. Because of the exponential attenuation of dust, $N^{*} \simeq N_{\mathrm{H}}^{\text {tor }}$ for $N>\sigma_{\text {FUv }}^{-1}$. It follows that

$$
I_{\mathrm{IR}} \propto \frac{d N_{\mathrm{H}}^{\mathrm{tot}}}{d t} \propto \frac{d N^{*}}{d t} \propto \int I_{0} G_{0} e^{-\sigma \mathrm{FuvN}} f\left(N_{\mathrm{H}_{2}}\right) n_{\mathrm{H}_{2}} d l .
$$

The sharp dissociation front makes the term $n_{\mathrm{H}_{2}} e^{-\sigma \mathrm{Fuv} N}$ peak sharply between $N^{*}$ and $N^{*}+\sigma_{\text {FUv }}^{-1}$ so that

$$
\frac{d N^{*}}{d t} \propto I_{0} G_{0} e^{-\sigma_{\mathrm{FUYN}} *}
$$

with the solution

$$
I_{\mathrm{IR}} \propto \frac{d N^{*}}{d t} \propto\left(I_{0} G_{0} t\right)^{-1}
$$

for the case $G_{0} / n \gtrsim 10^{-2} \mathrm{~cm}^{3}$ and $t_{\sigma} \lesssim t \lesssim t_{\text {eq }}$.

Finally, we consider the pumping rate in equilibrium, $t \gtrsim$ $t_{\text {eq. }}$. GS95 show that in this case $I_{\mathrm{IR}} \propto N_{\mathrm{H}}^{\mathrm{tot}} \sim \sigma_{\mathrm{FUV}}^{-1}$, ignoring a logarithmic term of order unity. This can be understood by noting that in equilibrium there are nine FUV pumps per $\mathrm{H}_{2}$ formation and that the total column formation rate of $\mathrm{H}_{2}$ is $R_{f} n N_{\mathrm{H}}^{\text {tot }}$. Thus, we obtain

$$
\begin{aligned}
I_{\mathrm{IR}}=I_{\mathrm{eq}} & \simeq \frac{9 R_{f} n N_{\mathrm{H}}^{\mathrm{tot}} \Delta E_{p}}{4 \pi} \\
& \simeq 9 \times 10^{-3} n_{5}\left(\frac{N_{\mathrm{H}}^{\mathrm{tot}}}{10^{21} \mathrm{~cm}^{-2}}\right) \mathrm{ergs} \mathrm{cm}^{-2} \mathrm{~s}^{-1} \mathrm{sr}^{-1} .
\end{aligned}
$$

To summarize these results for $n<n_{\mathrm{cr}}$ : if $G_{0} / n \lesssim 10^{-2} \mathrm{~cm}^{3}$, then $I_{\mathrm{IR}}=I_{\sigma}$, with $I_{\sigma}$ given by equation (13). If $G_{0} / n \gtrsim 10^{-2}$ $\mathrm{cm}^{3}$, then $I_{\mathrm{IR}}=I_{\sigma}$ for $t<t_{\sigma}, I_{\mathrm{IR}} \simeq I_{\sigma}\left(t_{\sigma} / t\right)$ for $t_{\sigma}<t<t_{\mathrm{eq}}$, and $I_{\mathrm{IR}} \simeq I_{\mathrm{eq}}$ for $t>t_{\mathrm{eq}}$, with $t_{\mathrm{eq}}$ given by equation (2), $t_{\sigma}$ by equation (10), and $I_{\text {eq }}$ by equation (17).

Figure 3 illustrates these low-density results for $G_{0} / n \gtrsim 10^{-2}$ $\mathrm{cm}^{3}$. The cumulative $\mathrm{H}_{2}$ intensity (the intensity produced by gas between 0 and $N$ ) is plotted as a function of $N$ at various times. The low-density case $n=10^{3} \mathrm{~cm}^{-3}$ is plotted so that the results are not complicated by the effects of collisional deexcitation. For $t<t_{\sigma} \sim 3 \times 10^{3} \mathrm{yr}$, note that most of the intensity is produced by gas at $N \sim 10^{21} \mathrm{~cm}^{-2}$ and that the total integrated intensity is constant in time. At later times, $t_{\sigma}<t<$ 


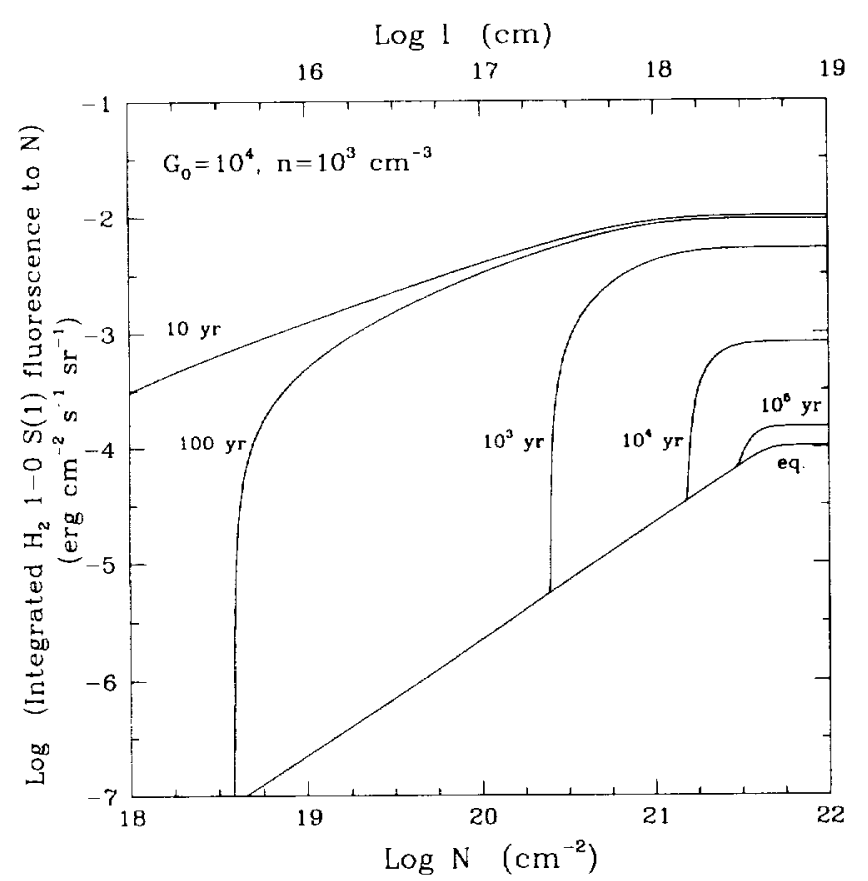

FlG. 3.- Cumulative fluorescent $1-0 S(1) \mathrm{H}_{2}$ intensity (i.e., the intensity produced by gas between 0 and $N$ ) shown as a function of the gas column density $N$ at various times for a case where $G_{0}=10^{4}, n=10^{3} \mathrm{~cm}^{-3}$. The figure shows that at early times most of the intensity is produced at $N \sim \sigma_{\mathrm{fuV}}^{-1} \sim 10^{21}$ $\mathrm{cm}^{-2}$.

$t_{\mathrm{eq}} \sim 5 \times 10^{5} \mathrm{yr}$, the intensity is produced somewhat deeper into the slab at the (attenuated) dissociation front, and $I_{\mathrm{IR}} \propto$ $t^{-1}$. Finally, in equlibrium, the intensity is mainly produced in the final logarithmic interval of $\Delta N$, where $\mathrm{H}$ rapidly converts to $\mathrm{H}_{2}$ (around $N \sim 3 \times 10^{21} \mathrm{~cm}^{-2}$ ).

At high density, $n>n_{\mathrm{cr}}$, the FUV fluorescent component of the $\mathrm{H}_{2}$ emission is reduced by a factor $n / n_{\mathrm{cr}}$. This energy goes into heat, and Figures 1 and 2 show that that this can raise the temperature of considerable column densities of $\mathrm{H}_{2}$ to $T>1000 \mathrm{~K}$. In this case $\mathrm{H}_{2}$ can act both as the dominant heat source and as the dominant coolant. The heat is delivered by the collisional de-excitation of FUV-pumped vibrational levels. The cooling arises from the collisional excitation, and subsequent radiative decay, of low-lying vibrational levels. Therefore, much of the fluorescent pump energy emerges as thermal emission from low-lying $\mathrm{H}_{2}$ vibrational transitions. To add to the complication, $n_{c r}$ is temperature sensitive and is smaller at higher temperatures, thereby enhancing collisional de-excitation.

\subsection{Model Results of Line Intensities}

Figures 4,5 , and 6 show the main results of significance to observers. They plot the total (thermal plus fluorescent) emergent intensity in the $\mathrm{H}_{2} 1-0 \mathrm{~S}(1)$ line, the fluorescent component of the $\mathrm{H}_{2} \quad 1-0 \mathrm{~S}(1)$ line, and the ratio of the total 2-1 $S(1) / 1-0 S(1)$ line intensities. Figure 4 demonstrates the dependences discussed above. For low values of $G_{0} / n$, less than $10^{-2}$ $\mathrm{cm}^{3}$, there is no time dependence of $I_{\mathrm{IR}} ; I_{\mathrm{IR}} \simeq I_{\sigma}$. On the other hand, for low values of $n, n<n_{\mathrm{cr}}$, and high values of $G_{0} / n$, greater than $10^{-2} \mathrm{~cm}^{3}$, we see the following sequence for $I_{\mathrm{IR}}(t)$ : first $I_{\sigma}$, then $I_{\sigma}\left(t_{\sigma} / t\right)$, and finally $I_{\text {eq }}$. The main result shown in Figure 4 is that the $\mathrm{H}_{2}$ intensities can show strong, timedependent variations to times as long as $5 \times 10^{8} / n$ yr. The nonequilibrium intensities can be at least an order of magnitude larger than the equilibrium values.

Figure 5 shows the effects of collisional de-excitation of the pump-excited vibrational levels. At low density, $n=10^{3} \mathrm{~cm}^{-3}$, $2-1 S(1) / 1-0 \quad S(1) \sim 0.5$, the pure-fluorescent value (with a slight variation at $t=100$ yr for $G_{0} \gtrless 10^{5}$ due to the temperature dependence of $n_{\mathrm{cr}}$ ). There is also a pure-fluorescent ratio for densities as high as $10^{6} \mathrm{~cm}^{-3}$ if $G_{0} \leqslant 10^{3}$ since the pump region is cold and the critical densities are high $\left(\geq 10^{6}\right.$ $\mathrm{cm}^{-3}$ ) for such cold temperatures. However, for $G_{0} \gtrsim 10^{4}$ and $n \gtrsim 10^{3}-10^{4} \mathrm{~cm}^{-3}$, the $2-1 \quad S(1) / 1-0 \quad S(1)$ ratio rapidly approaches a thermal value of order 0.1 . This ratio has often been interpreted to indicate shock emission, but Figure 5 demonstrates that collisionally altered FUV pumping can produce this ratio. Figure 5 also shows that the timescale for the $2-1 S(1) / 1-0 S(1)$ ratio to reach a steady state is somewhat less than $t_{\mathrm{eq}}$.

Figure 4 and 6 also show the effects of collisional deexcitation of FUV-pumped $\mathrm{H}_{2}$. Figure 6 plots the fluorescent component of the 1-0 S(1) intensity in order to provide an estimate of the intensities of the higher vibrational lines $(v>2)$. The higher vibrational states will not have a significant thermal component because they lie $\Delta E / k \gtrsim 18,000 \mathrm{~K}$ above the ground state and cannot be significantly excited by collisions with gas at $T \sim 1000 \mathrm{~K}$. Therefore, their intensities can be estimated from Figure 6 by using the pure-fluorescent ratios of the lines in question to 1-0 S(1) (see, e.g., Sternberg 1988) and multiplying the ratio by the 1-0 S(1) intensity in Figure 6.

Figure 7 plots the time-dependent intensities in the strong (surface brightness greater than $10^{-6} \mathrm{ergs} \mathrm{cm}^{-2} \mathrm{~s}^{-1} \mathrm{sr}^{-1}$ ) coolants $\mathrm{OH}, \mathrm{H}_{2} \mathrm{O}, \mathrm{Fe}$ II $1.64 \mu \mathrm{m}, \mathrm{O}$ I $6300 \AA$, and $\mathrm{S}$ II $6730 \AA$, which show some interesting dependences. Individual lines from these coolants have surface brightnesses greater than $10^{-6}$ ergs cm $\mathrm{cm}^{-2} \mathrm{~s}^{-1} \mathrm{sr}^{-1}$ only for the case $n=10^{6} \mathrm{~cm}^{-3}$, so we have only plotted this case. Because only the high-density cases have detectable lines, steady state is rapidly reached in 10-100 yr. The strong lines of C II $158 \mu \mathrm{m}, \mathrm{O}$ I $63 \mu \mathrm{m}$, and Si II $35 \mu \mathrm{m}$ are not shown because they show little time dependence and steady state values can be taken from TH85 and HTT91. Therefore, Figure 7 demonstrates that the emission from other species will probably not help diagnose nonequilibrium in time-dependent PDRs. Observers can take the values from equilibrium PDR models to compare with observed line intensities.

\subsection{Model Results of the Evolution of the Atomic Hydrogen Column}

The $\mathrm{H}_{\mathrm{I}} 21 \mathrm{~cm}$ line can be used to trace the total atomic hydrogen column $N_{\mathrm{H}}^{\text {tot }}$ in planetary nebulae and other potentially time-dependent PDRs. Likkel et al. (1992) present upper limits to the $21 \mathrm{~cm}$ fluxes from two compact planetary nebulae $\left(\mathrm{BD}+30^{\circ} 3639\right.$ and $\left.\mathrm{AFGL} 618\right)$ and review previous $21 \mathrm{~cm}$ studies of planetary nebulae. Taylor, Gussie, \& Pottasch (1990) have observed $\mathrm{H}, 21 \mathrm{~cm}$ in a few planetary nebulae and conclude that the $\mathrm{H}$ I mass detected cannot adequately account for the mass expected to have been lost by the asymptotic giant branch precursor star. It is possible the mass is in $\mathrm{H}_{2}$ and that the dissociation front has not yet propagated through the bulk of the mass.

Figure 8 therefore plots $N_{\mathrm{H}}^{\text {tot }}$ as a function of $n, G_{0}$, and $t$. The time-dependent creation of atomic hydrogen is clearly seen, and the final equilibrium columns are generally of order $10^{21}$ $\mathrm{cm}^{-2}$ because dust attenuation of the FUV preserves the 

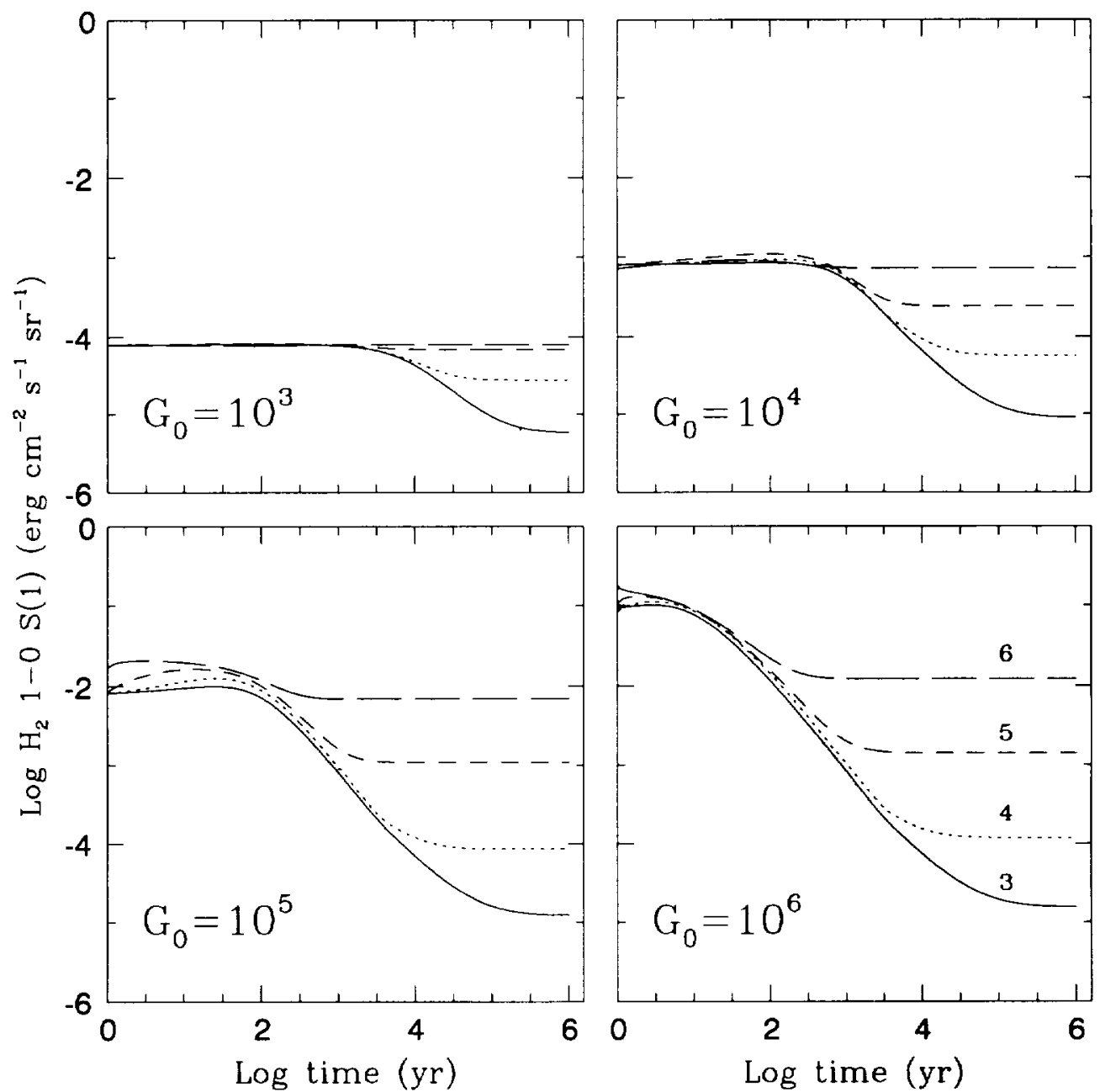

Fic. 4.-Total intensity of the $\mathrm{H}_{2} \mathrm{l}-\mathrm{OS}(1)$ line as a function of time; the units are ergs $\mathrm{cm}^{-2} \mathrm{~s}^{-1} \mathrm{sr}^{-1}$. Each panel refers to a different value of $G_{0}$. as labeled. The solid lines are for $n=10^{3} \mathrm{~cm}^{-3}$, the dotted lines for $n=10^{4} \mathrm{~cm}^{-3}$, the short-dashed lines for $n=10^{5} \mathrm{~cm}^{-3}$, and the long-dashed lines for $n=10^{6} \mathrm{~cm}^{-3}$. In the $G_{0}=10^{6}$ panel, $\log n$ is explicitly labeled on the lines. For $G_{0} n>10^{-2} \mathrm{~cm}^{3}$, the intensity drops with time from a peak value $I_{\sigma}$ to $I_{\text {eq }}$ (see text).

hydrogen molecules at higher columns. For low values of $G_{0} / n$, $\leqslant 10^{-2} \mathrm{~cm}^{3}$, the equilibrium values of $N_{\mathrm{H}}^{\text {tor }}$ are significantly smaller than $10^{21} \mathrm{~cm}^{-2}$ because of $\mathrm{H}_{2}$ self-shielding.

\section{APPLICATIONS}

GS95 discuss applications of time-dependent PDR models to reflection nebulae and to neutral gas in starburst galaxy nuclei or in active galactic nuclei. They also discuss application to planetary nebulae (PNs), and we amplify that discussion here since PNs are our primary motivation in developing this code. The neutral densities in PNs often exceed $10^{4} \mathrm{~cm}^{-3}$ (otherwise the gas is rapidly ionized), and the timescales for changes in the FUV flux and gas density are short, of order 100-1000 yr. The PDR region lies in a thin shell of thickness $\Delta R$ outside of the ionized nebula at a distance $R$ from the central white dwarf. Because $\Delta R / R \ll 1$, the one-dimensional slab models well approximate the conditions in the PDRs in PNs (Natta \& Hollenbach 1995).

Huggins $(1992,1993)$ reviews the status of observations of neutral gas associated with PNs. Over 33 PNs have been detected in $\mathrm{H}_{2} 2 \mu \mathrm{m}$ vibrational emission. Although purefluorescent emission has been observed (for example, in
Hubble 12 and $\mathrm{BD}+30^{\circ} 3639$; Dinerstein et al. 1988), in many cases the 2-1 $S(1) / 1-0 S(1)$ ratio is of order $\sim 0.1$ (e.g., Zuckerman \& Gatley 1988). This has often been interpreted as arising from shock emission, perhaps caused by the ejection of a dense shell that supersonically overtakes the slowly expanding red giant wind of the PN progenitor. However, as has been already argued (Hollenbach 1988; Sternberg \& Dalgarno 1989; Burton et al. 1990), FUV pumping at high density can produce similar "thermal" ratios.

Without detailed models of individual PNs (which is the subject of Natta \& Hollenbach 1995), it is difficult to argue that the observed $\mathrm{H}_{2}$ emission may reflect FUV pumping of nonequilibrium $\mathrm{H}_{2}$, even if the incident FUV flux can be estimated. As Figure 4 demonstrates, for fixed $G_{0}$, a given intensity can be produced at early times in low-density, nonequilibrium PDRs or at late times from equilibrium PDRs of higher density. GS95 argue that the densities in the planetaries NGC 7027 and BD $+30^{\circ} 3639$ are sufficiently low $\left(\leqq 3 \times 10^{4} \mathrm{~cm}^{-3}\right)$ that nonequilibrium models are needed to explain the observed intensities. However, in the case of NGC 7027, the 2-1 S(1)/1-0 S(1) ratio is low, $\sim 0.07$, and not the $\sim 0.5$ expected from the pure fluorescence of a low-density PDR (Tanaka et al. 1989). In addi- 

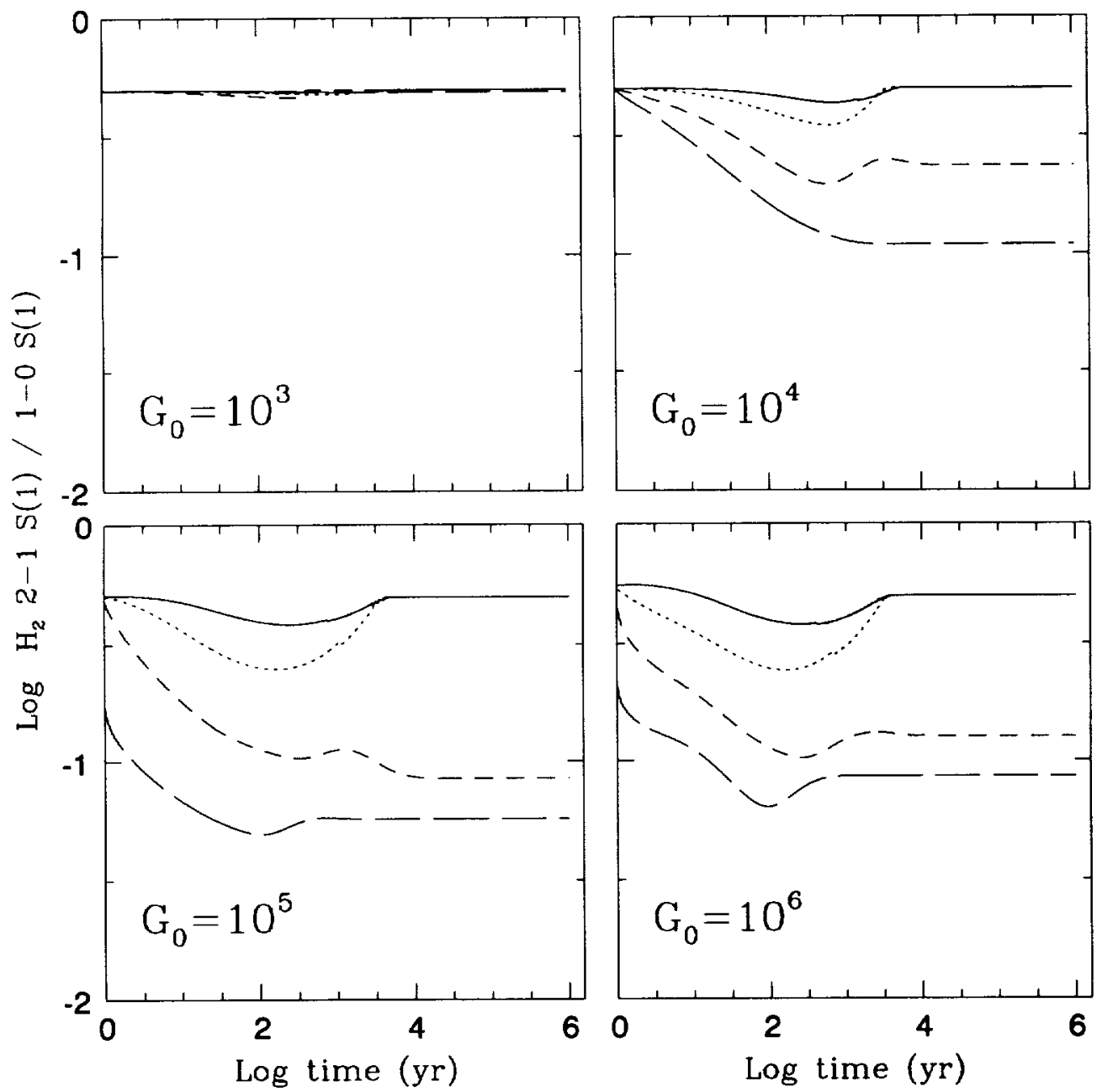

Fig. 5.-Ratio of the 2-1 $S(1)$ to the 1-0 S(1) line intensities as a function of time. Lines denote values of $n$ as in Fig. 4 . A ratio of 0.5 indicates pure fluorescence; lower ratios indicate contributions from thermal collisions.

tion, a PDR model fit to the $\mathrm{O}_{\text {I }} 63 \mu \mathrm{m}$ and $\mathrm{C}$ II $158 \mu \mathrm{m}$ intensities predicts PDR densities of $7 \times 10^{4} \mathrm{~cm}^{-3}$ (Ellis \& Werner 1985) and an FUV flux of $G_{0}=4 \times 10^{4}$ (GS95). The peak 1-0 $S(1)$ intensity is about $1 \times 10^{-3} \mathrm{ergs} \mathrm{cm}^{-2} \mathrm{~s}^{-1} \mathrm{sr}^{-1}$ (Graham et al. 1993a, b; Cox et al. 1995). Figures 4 and 5 show that a reasonable fit to the $1-0 S(1)$ and the $2-1 S(1)$ line intensities can be obtained if $G_{0} \sim 10^{5}$ and $n \sim 10^{5} \mathrm{~cm}^{-3}$, consistent with the fine-structure lines. However, the fit requires $t \gtrsim 10^{3}$ yr and nearly equilibrium conditions. Therefore, NGC 7027 may not show evidence of nonequilibrium chemistry.

$\mathrm{BD}+30^{\circ} 3639$ is a better case because the spectrum looks like pure fluorescence, which suggests low densities, $n \leqslant 10^{4}$ $\mathrm{cm}^{-3}$, yet the 1-0 $S(1)$ peak intensity is also about $1 \times 10^{-3}$ ergs $\mathrm{cm}^{-2} \mathrm{~s}^{-1} \mathrm{sr}^{-1}$ (Graham et al. 1993a). GS95 quote $G_{0} \sim 2$ $\times 10^{4}$. Figures 4 and 5 show that a fluorescent $2-1 S(1) / 1-0$ $S(1)$ ratio of 0.5 and the observed $1-0 S(1)$ intensity are produced if $n \lesssim 10^{4} \mathrm{~cm}^{-3}$ and if $t \leqslant 10^{3} \mathrm{yr}$. The nonequilibrium $\mathrm{H}_{2}$ intensity in this case is at least of an order of magnitude higher than the equilibrium value.

Zuckerman \& Gatley (1988) observed $\mathrm{H}_{2}$ emission in three planetaries, NGC 2346, NGC 6720 (the Ring), and NGC 6853 (the Dumbbell). The peak 1-0 $S(1)$ intensities are about
$1 \times 10^{-4}$ ergs cm $\mathrm{cm}^{-2} \mathrm{~s}^{-1} \mathrm{sr}^{-1}$ and the inferred $G_{0} \lesssim 10^{3}$ in all three cases. The 2-1 $S(1) / 1-0 S(1)$ ratios are all less than 0.1 . The neutral densities are poorly constrained, but an estimate can be made with the assumption that the neutral gas is in pressure equilibrium with the ionized gas. Zuckerman \& Gatley estimate electron densities in the ionized gas of 300 $1000 \mathrm{~cm}^{-3}$, which indicate neutral densities of $n \sim 10^{4} \mathrm{~cm}^{-3}$. They argue that shock emission, with $\mathrm{H}_{2}$ excited by collisions with other hydrogen molecules, will be too weak to explain the observed intensities, so a PDR model is worth considering. Figure 4 shows that the observed peak intensities can (barely) be obtained in nonequilibrium models, if one assumes the most optimistic (high) values of $G_{0}$. If the neutral densities are $\lesssim 10^{4}$ $\mathrm{cm}^{-3}$, then nonequilibrium models apply and $t \leqslant 10^{4}$ yr is required. However, Figure 5 predicts that all models with $G_{0} \lesssim 10^{3}$ and $n \lesssim 10^{6} \mathrm{~cm}^{-3}$ have fluorescent ratios $2-1 S(1) /$ $1-0 S(1) \sim 0.5$, contrary to the observations. We conclude that even a time-dependent PDR model has difficulty explaining the observations. Perhaps the best solution at this time is one proposed by Zuckerman \& Gatley: the $\mathrm{H}_{2}$ is excited by collisions with hydrogen atoms (which have much larger rate coefficients than hydrogen molecules) in shock waves. 

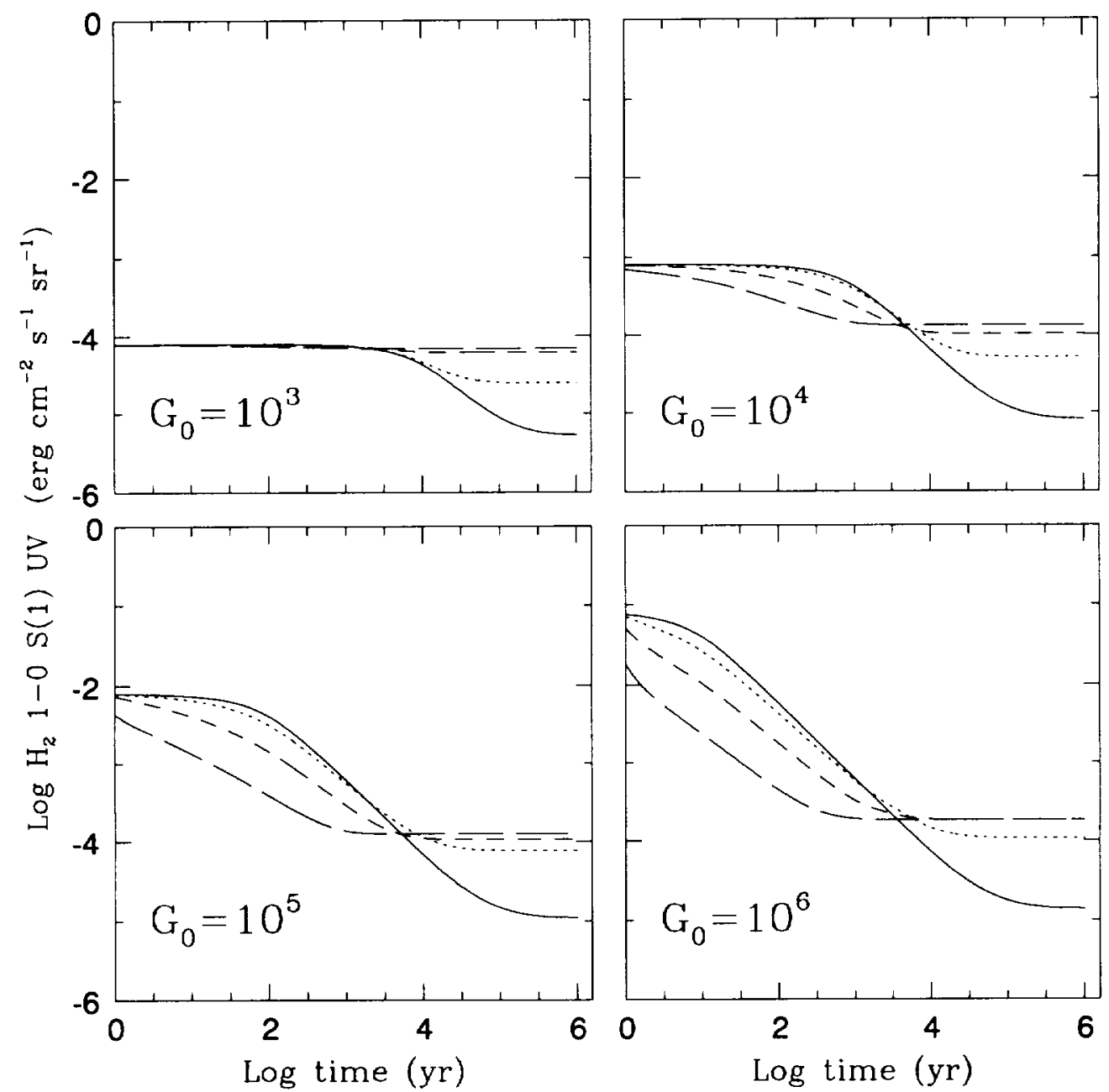

Fic. 6-Same as Fig. 4, but for the fluorescent component of the $1-0 \mathrm{~S}(1)$ line. This intensity may be multiplied by the pure-fluorescent ratios of high vibrational (v) lines to 1-0 S(1) as in, e.g., Sternberg (1988) to obtain predictions on the time-dependent intensities from high-1 lines that have no thermal component.

\section{SUMMARY AND CONCLUSION}

In this paper the time dependence of photodissociation regions was examined specifically for the case of a fully molecular slab that is suddenly exposed to an FUV flux $G_{0}$. Our time-dependent PDR code follows the photodissociation and fluorescence of $\mathrm{H}_{2}$ and includes the calculation of the heating that accompanies the rapid FUV pumping and dissociation of the hydrogen molecule. Both thermal collisions and fluorescence contribute to the excitation of $\mathrm{H}_{2}$ vibrational lines. If $G_{0} \gtrsim 10^{4}$ and $n \gtrsim 10^{5} \mathrm{~cm}^{-3}$, thermal collisions dominate the $1-0 S(1)$ and 2-1 $S(1)$ line intensities at all times; thermal collisions contribute significantly even for densities as low as $n \sim 10^{4} \mathrm{~cm}^{-3}$ if $t \leqq 10^{3} \mathrm{yr}$. These thermal collisions produce $2-1 S(1) / 1-0 S(1)$ ratios $\lesssim 0.1$. Such ratios have often been taken to indicate shock excitation; however, in this case it is simply FUV-heated gas. Figures 4,5 , and 6 present a parameter study in $n, G_{0}$, and $t$ of the 1-0 S(1) and 2-1 S(1) line intensities and a method to calculate the emergent intensities of higher vibrational (pure fluorescent) transitions.

As the dissociation wave sweeps into the slab, the $\mathrm{H}_{2}$ vibrational intensities are time dependent only if $G_{O} / n>10^{-2} \mathrm{~cm}^{3}$. In this case, the time dependence proceeds as follows: initially, for $t<t_{\sigma}$ (see eq. [10]), the intensity has a constant value of $I_{\sigma}$ (eq. [13]); for $t_{\sigma}<t<t_{\text {eq }}$ (eq. [2]), the intensity drops as $I_{\sigma}\left(t_{\sigma} / t\right)$; finally, for $t>t_{\mathrm{eq}}$, the intensity asymptotically approaches the constant $I_{\mathrm{eq}}$ (eq. [17]). The dissociation wave is characterized by a steep "front," which initially accelerates through the slab with velocity proportional to time. The front slows once it penetrates to a column density $N \sim \sigma_{\mathrm{FuV}} \sim 10^{21}$ $\mathrm{cm}^{-2}$, where dust attenuation begins to sharply reduce the dissociating-photon flux. At all times, most of the $\mathrm{H}_{2}$ vibrational-line intensities are produced at $N \sim 10^{21} \mathrm{~cm}^{-2}$, and it is the drop in $\mathrm{H}_{2}$ abundance at this column density that causes the $\mathrm{H}_{2}$ intensity to drop. Our calculations extend to higher density the recent results of GS95 for the fluorescent $\mathrm{H}_{2}$ emission.

We also presented (Figure 7) the surface brightnesses of $\mathrm{O} I$ $6300 \AA, S$ II $6730 \AA$, Fe II $1.64 \mu \mathrm{m}$, and the total rotational contributions of $\mathrm{OH}$ and $\mathrm{H}_{2} \mathrm{O}$. The intensities of these transitions also show interesting time dependence and are observable if $n \gtrsim 10^{6} \mathrm{~cm}^{-3}$. The atomic lines also require $G_{0} \gtrsim 10^{5}$ for observability. The total atomic hydrogen column densities are shown in Figure 8 for possible application to $\mathrm{H} 121 \mathrm{~cm}$ observations. 

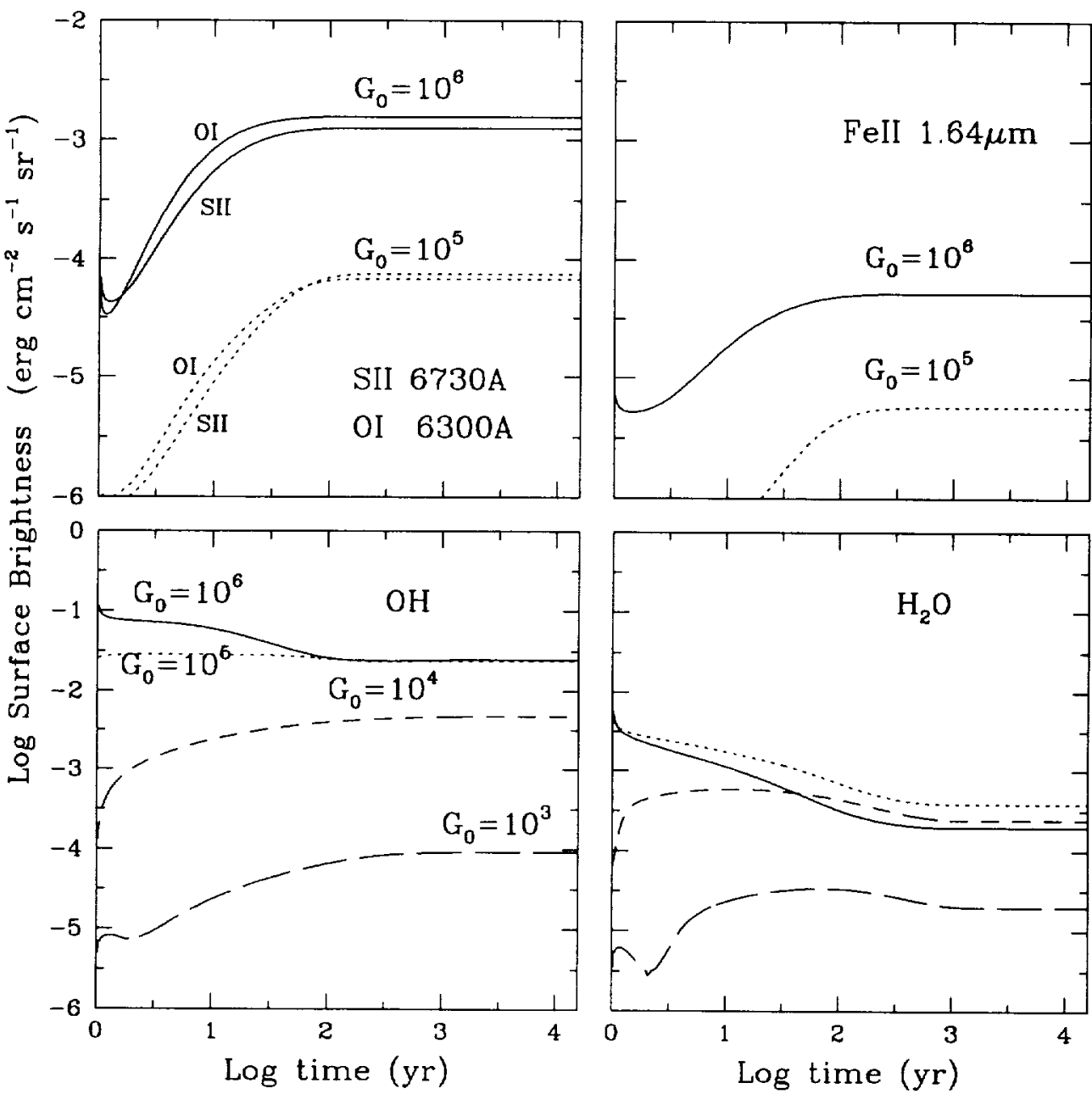

Fig. 7. - Intensity of some metal and molecular lines as a function of time. Long-dashed lines refer to models with $G_{0}=10^{3}$, short-dashed lines to $G_{0}=10^{4}$, dotted lines to $G_{0}=10^{5}$, and solid lines to $G_{0}=10^{6}$. In all cases, $n=10^{6} \mathrm{~cm}^{-3}$. The $\mathrm{OH}$ and $\mathrm{H}_{2} \mathrm{O}$ panels refer to total intensities in all pure-rotational lines of $v=0$.

We applied these models to $\mathrm{H}_{2}$ observations of five planetary nebulae and found that $\mathrm{BD}+30^{\circ} 3639$ may show evidence of early time-enhanced intensities, whereas the $\mathrm{H}_{2}$ intensities in NGC 7027, NGC 2346, NGC 6720, and NGC 6853 are better explained by equilibrium PDR models or shock excitation.
We acknowledge useful discussions with A. Sternberg, $O$. Goldshmidt, and A. Tielens. D. J. H. thanks the NASA Astrophysical Theory Program for support under NASA RTOP 399-20-10-12. This work was also supported by the ASI (Italian Space Agency), grants 92-RS-54 and 94-RS-152.

\section{APPENDIX}

\section{NUMERICAL METHOD}

We assume a constant-density, semi-infinite slab, initially fully molecular and then illuminated at time $t=0$ by an FUV flux $G_{0}$. Although the temperature varies in the slab, we assume that turbulence dominates the pressure so that the density is always constant and uniform (as did the original equilibrium PDR models of TH85). The code numerically integrates equation (1), using logarithmic intervals of hydrogen nucleus column density and time. The initial column density bin has a column density $\Delta N=10^{17}$ $\mathrm{cm}^{-2}$, and the initial time step is $\Delta t=1 \mathrm{yr}$. Thereafter, we use typically 200 column density bins per decade and 100 timesteps per decade. We have reduced the bin sizes and the time steps by factors of 2 until successive solutions match to better than a few percent. We have also compared the results with analytic solutions for the time dependence (see $\S 3$ ) and the equilibrium solution. Finally, we have run several cases with constant temperature and low density to compare the results with the values quoted by GS95. We find good agreement (differences of less than $\pm 30 \%$, and even less when we adjust for the slightly different values of $I_{0}$ and $\sigma_{\mathrm{Fuv}}$ in our code).

The only difficulty in this integration is the large difference between the timescale for dissociation in the unshielded FUV field, $t_{\min } \sim\left(10^{3} / G_{0}\right) \mathrm{yr}$, compared to the timescale to reach final equilibrium, $t_{\mathrm{eq}} \sim\left(5 \times 10^{8} / n\right) \mathrm{yr}$. If we are forced to take time steps of 

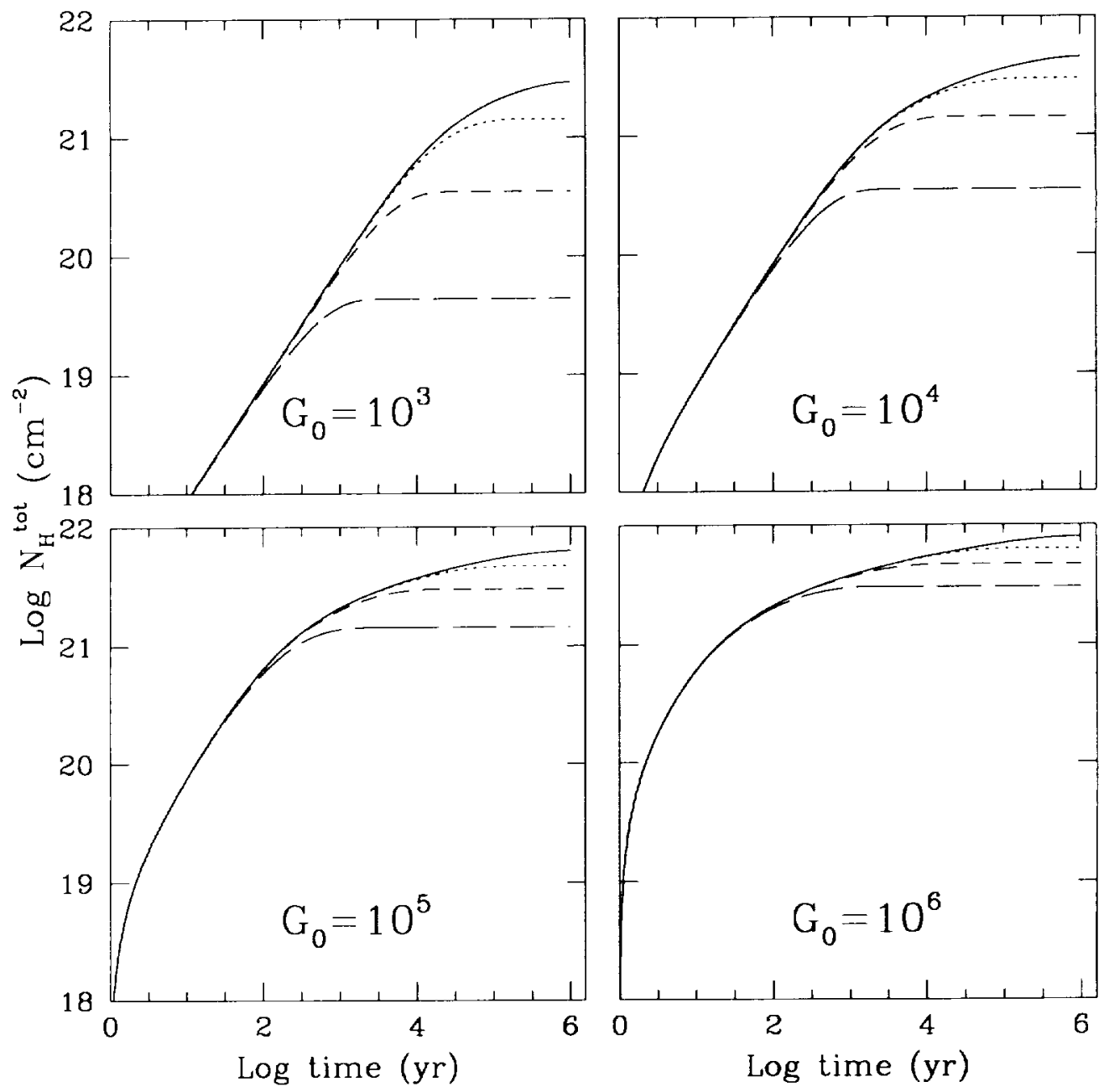

FiG. 8.-Total atomic hydrogen column density $N_{\mathrm{H}}^{\text {tot }}$ plotted as a function of $t, G_{0}$, and $n$. The different lines denote different densities; lines denote values of $n$ as in Fig. 4.

less than $t_{\min }$, then we would be forced to take $10^{8}$ time steps (on each of 1000 spatial grid points) in order to follow the evolution of the case $n=10^{3} \mathrm{~cm}^{-3}$ and $G_{0}=10^{5}$, for example! We therefore use the following algorithm, which allows time steps much greater than $t_{\text {min }}$. We integrate equation (7) over a small time step $\Delta t$ and find, at a column density $N$,

$$
x_{\mathrm{H}_{2}}(t+\Delta t)=\left[x_{\mathrm{H}_{2}}(t)-x_{\mathrm{eq}}\right] e^{-\Delta t / t_{\mathrm{ch}}}+x_{\mathrm{eq}},
$$

where $x_{\mathrm{H}_{2}}$ is the $\mathrm{H}_{2}$ abundance and where

$$
x_{\mathrm{eq}}=R_{f} n t_{\mathrm{ch}}
$$

is the equilibrium abundance of $\mathrm{H}_{2}$,

$$
t_{\mathrm{ch}}=\left[2 R_{f} n+G_{0} I_{0} f\left(N_{\mathrm{H}_{2}}^{*}\right) e^{-\sigma_{\mathrm{Fuv} N}}\right]^{-1}
$$

is the characteristic timescale for change of the $\mathrm{H}_{2}$ abundance, and

$$
N_{\mathbf{H}_{2}}^{*}=\left[N_{\mathrm{H}_{2}}(t)+N_{\mathbf{H}_{2}}(t+\Delta t)\right] / 2
$$

is the average shielding column density between $t$ and $t+\Delta t$. The column density $N_{\mathrm{H}_{2}}(t+\Delta t)$ is known because we start at the surface and solve for $x_{\mathrm{H}_{2}}(t+\Delta t)$ from the surface into column density $N$. With this method, even if $\Delta t \gg t_{\mathrm{ch}} \geq t_{\mathrm{min}}$, the solution moves in an appropriate way toward the equilibrium solution. 


\section{REFERENCES}

Bakes, E. L. O., \& Tielens, A. G. G. M. 1994, ApJ, 427, 822

Burton, M. G., Hollenbach, D. J., \& Tielens, A. G. G. M. 1990, ApJ, 365, 620

Cox, P., Maillard, J.-P., Huggins, P. J., Forveille, T., Bachiller, R., Guilloteau, S., \& Omont, A. 1995, in IAU Colloq. 149, 3D-Spectroscopy, ed. G. Comte (Dordrecht: Kluwer), in press

Dinerstein, H. L., Lester, D. F., Carr, J. S., \& Harvey, P. M. 1988, ApJ, 327, L27 Ellis, H. B., \& Werner, M. W. 1985, in Mass Loss from Red Giants, ed. M. Morris \& B. Zuckerman (Dordrecht: Reidel), 309

Genzel, R. 1991, in The Physics of Star Formation and Early Stellar Evolution, ed. C. Lada \& N. Kylafis (Dordrecht: Kluwer), 155

1992, in The Galactic Interstellar Medium: Saas Fée Lectures 1991, ed. W. B. Burton, B. G. Elmegreen, \& R. Genzel (New York: Springer), 275 Goldshmidt, O., \& Sternberg, A. 1995, ApJ, 439, 256

Graham, J. R., Herbst, T., Matthews, K., Neugebauer, G., Soifer, B. T., Serabyn, E., \& Beck with, S. 1993a, ApJ, 408, L105

Graham, J. R., Serabyn, E., Herbst, T., Beckwith, S., Matthews, K., Neugebauer, G., Soifer, B. T., \& Wilson, T. D. 1993b, AJ, 105, 250

Hill, J. K., \& Hollenbach, D. 1978, ApJ, 225, 390

Hollenbach, D. 1988, Astrophys. Lett. Commun., 26, 191

Hollenbach, D., \& McKee, C. F. 1979, ApJS, 41, 555

$-1989, \mathrm{ApJ}, 342,306$
Hollenbach, D., Takahashi, T., \& Tielens, A. G. G. M. 1991, ApJ, 377, 192

Hollenbach, D., \& Tielens, A. G. G. M. 1995 , in Lecture Notes in Physics, 459 The Physics and Chemistry of Interstellar Molecular Clouds, ed. G. Winnewisser \& G. C. Petz (New York: Springer), in press

Huggins, P. J. 1992, in IAU Symp. 155, Planetary Nebulae, ed. R. Weinberger \& A. Acker (Dordrecht : Kluwer), 147 1993, in Mass Loss on the AGB and Beyond, ed. H. Schwarz (Garching: ESO), 365

Jura, M. 1974, ApJ, 191, 375

Likkel, L., Gussie, G. T., Taylor, A. R., \& Dewdney, P. E. 1992, AJ, 103, 538

London, R. 1978, ApJ, 225, 405

Natta, A., \& Hollenbach, D. 1995 in preparation

Roger, R. S., \& Dewdney, P. E. 1992, ApJ, 385, 536

Sternberg, A. 1988, ApJ, 332, 400

Sternberg, A., \& Dalgarno, A. 1989, ApJ, 338, 197

Tanaka, M., Hasegawa, T., Hayashi, S. S., Brand, P. W. J. L., \& Gatley, I. 1989, ApJ, 336, 207

Taylor, A. R., Gussie, G. T., \& Potasch, S. R. 1990, ApJ, 351, 515

Tielens, A. G. G. M., \& Hollenbach, D. 1985, ApJ, 291, 722

van Dishoeck, E. F., \& Black, J. H. 1988, ApJ, 334, 771

Zuckerman, B., \& Gatley, I. 1988, ApJ, 324, 501 